\title{
A Target Tracking Method to Reduce the Energy Consumption in Wireless Sensor Networks
}

\author{
Hyunsook Kim and Kijun Han ${ }^{*}$ \\ Department of Computer Engineering, \\ Kyungpook National University, Daegu, Korea \\ hskim@netopia.knu.ac.kr, kjhan@knu.ac.kr
}

\begin{abstract}
Reducing the energy consumption is one of the most critical issues in wireless sensor networks and an accurate prediction is especially required in tracking. It is desirable that only the nodes surrounding the mobile target should be responsible for observing the target to save the energy consumption and extend the network lifetime as well. In this paper, we propose an efficient tracking method based on prediction through the error correction, which can minimize the number of participating sensor nodes for target tracking. We show that our tracking method performs well in terms of saving energy regardless of mobility pattern of the mobile target.
\end{abstract}

\section{Introduction}

Object tracking is emerging as one of the new attractive applications in large-scale wireless sensor networks such as wild animal habit monitoring and intruder surveillance in military regions. Tracking of the mobile targets has lots of open problems to be solved including target detection, localization, data gathering, and prediction.

In the localization problem, excessive sensors may join in detection and tracking for only a few targets. And, if all nodes have to always wake up to detect a mobile target, there are a lot of waste of resources such as battery power and channel utilization. So, if each node uses timely its energy to execute tasks, the network lifetime may be extended as a whole. This raises the necessity for prediction of the moving path of the mobile target to maintain the number of participating nodes in tracking as small as possible.

Many tracking protocols in large-scale sensor networks have been proposed to solve the problems concerned with tracking of the mobile targets from various angles $[1,2,3,4]$.

In this paper, we present a tracking method by predicting the location of the mobile target in 2-dimensional WSN, based on linear estimation.

The rest of this paper is organized as follows. Our proposed tracking method is presented in Section 2. Next, in Section 3, we present some simulation results. Finally, Section 4 concludes the paper.

* Correspondent author. 


\section{Proposed Tracking Method}

\subsection{Approximate Prediction Step}

First, since we assume that the mobile target does not change its direction or speed so abruptly in the sensing field, the location of the mobile object at the time instant of $(\mathrm{n}+1)$ is approximately predicted by estimating the velocity when the mobile target will move during the time interval $[n, n+1]$. Fig.1 shows the concept of tracking of the mobile target.

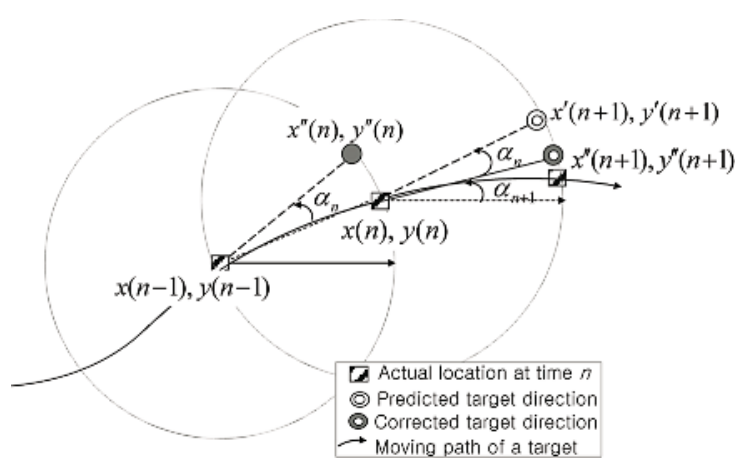

Fig. 1. Tracking of the mobile target in WSN

Given the current location $(x(n), y(n))$, the first predicted location of the mobile object at the time instant of $(\mathrm{n}+1)$, denoted by $\left(x^{\prime}(n+1), y^{\prime}(n+1)\right)$ is

$$
x^{\prime}(n+1)=x(n)+\widetilde{v}_{x}(n+1), \quad y^{\prime}(n+1)=y(n)+\tilde{v}_{y}(n+1)
$$

where $\widetilde{v}_{x}(n+1)$ and $\widetilde{v}_{y}(n+1)$ represent the future speed estimates of the mobile object in the direction of $x$ and $y$, respectively.

These speed estimates based on the previous history are given by

$$
\tilde{v}_{x}(n+1)=\frac{\sum_{i=n-h+1}^{n} \tilde{v}_{x}(i)}{h}, \quad \widetilde{v}_{y}(n+1)=\frac{\sum_{i=n-h+1}^{n} \tilde{v}_{y}(i)}{h},
$$

where $h$ is a predefined number of the past history based on which we predict the next moving factor. Hence, the future speed is a moving average of acceleration of an object. That is, the present movement of a mobile object means a reflection of the patterns of the moving history.

The estimate obtained in this step makes it possible to exactly predict the future location of the mobile object that moves linearly. However, the estimate is no longer effective when the mobile object moves in non-linear fashion since only velocity information in used to predict the future location. So, we need a correction mechanism to get a more exact estimate. 


\subsection{Correction Step}

Let us express the prediction error by the angle between the actual location and the previously predicted, denoted by $\alpha$. Then we have

$$
\cos \alpha_{n}=\frac{x^{\prime \prime}(n)-x(n-1)}{\sqrt{\left[x^{\prime \prime}(n)-x(n-1)\right]^{2}+\left[y^{\prime \prime}(n)-y(n-1)\right]^{2}}}-\frac{x(n)-x(n-1)}{\sqrt{[x(n)-x(n-1)]^{2}+[y(n)-y(n-1)]^{2}}}
$$

Then we can predict the new moving direction of the mobile target by

$$
\cos \alpha_{n+1}=\frac{x^{\prime}(n+1)-x(n)}{\sqrt{\left[x^{\prime}(n+1)-x(n)\right]^{2}+\left[y^{\prime}(n+1)-y(n)\right]^{2}}}-\cos \alpha_{n}
$$

Finally, we can predict the next location of the mobile target by correcting an angle from the first predicted location.

Tracking in our system is performed by the following procedure.

1. Discovery: When a sensor node around the mobile object detects the target and initializes tracking, it becomes 'estimation node' which acts as a master node temporarily.

2. Localization: A set of nodes those become aware the appearances of the mobile target compute the target's current position. The coordinates of the mobile target may be accomplished by the triangulation and their collaborative works.

3. Estimation: An estimation node predicts the future movement path of the mobile target, and transmits message about the approaching location to its neighbor nodes. The prediction is carried out by two steps: approximate a prediction and correction step that is explained above. The moving factors of a mobile target, such as direction and velocity, can be obtained by sensor nodes through collecting moving patterns of the tracked target.

4. Communication: As the mobile target moves, each node may hand off an initial estimate of the target location to the next node in turn. At that time, each node changes its duty cycle along the movement of the target.

\section{Simulation}

We carry out experiments to measure the missing rate and wasted energy.

To model the movement behavior of the mobile target, we use the Random Way Point model (RWP) and Gauss-Markov mobility model. Energy consumption used for simulation is based on some numeric parameters obtained in [2]. Our prediction method is compared with the Least Squares Minimization (LSQ) to evaluate the performance of accuracy. LSQ is a common method used for error reduction in estimation and prediction methods.

As shown in Fig. 2 (a), our scheme offers a smaller missing rate than LSQ regardless of mobility model used in simulation. In RWP, since the moving pattern of the mobile target is random, the prediction error increases. As the sensing range becomes larger, the missing rate decreases as well. Due to the inaccurate prediction of location, some nodes miss the target because the real location of the target is out of the sensing range. 


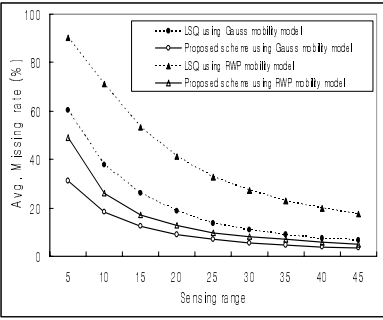

(a) Missing rate

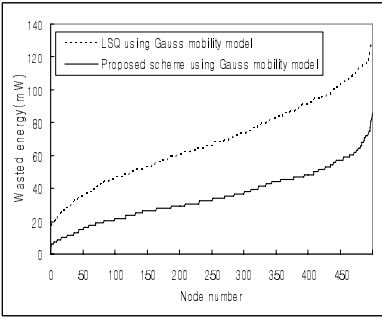

(b) Wasted energy in Gaussian Markov mobility

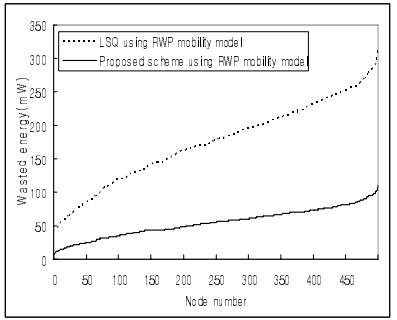

(c) Wasted energy in RWP

Fig. 2. Wasted energy

As shown Fig. 2(b) and 2(c), energy consumption is greatly influenced by an accuracy of prediction. As described earlier, we can extend the network lifetime by avoiding such unnecessary energy consumption at nodes that do not need to join in tracking. This figure indicates that our scheme can decrease the number of participating nodes and thus reduce the energy consumption too.

\section{Conclusion}

Power conservation and an accurate prediction are important issues for object tacking in wireless sensor networks. In this paper, we propose an efficient tracking method using a moving average estimator to decide the future location of the mobile target. And we improve the accuracy of prediction through the error correction. Simulations results show that our estimation method performs accurately, which contributes to saving energy and thus extending the network lifetime as well regardless of mobility pattern of the mobile target by reducing the number of participating nodes in tracking.

\section{Acknowledgement}

This research is supported by Program for the Training of Graduate Students for Regional Innovation.

\section{References}

1. C. Gui and P. Mohapatra. : Power conservation and quality of surveillance in target tracking sensor networks. In Proceeding of the ACM MobiCom, Philadelphia, PA, September (2004)

2. Y. Xu, J. Winter, and W.-C. Lee. : Prediction-based strategies for energy saving in object tracking sensor networks. In Proceedings of the Fifth IEEE International Conference on Mobile Data Management (MDM), USA, January (2004)

3. Y.Xu and W.C.Lee. : On Localized Prediction for Power Efficient Object Tracking in Sensor Networks. In 1st International Workshop on Mobile Distributed Computing, May (2003)

4. H. Yang and B. Sikdar. : A Protocol for Tracking Mobile Targets using Sensor Networks. In IEEE International Workshop on Sensor Network Protocols and Applications, May (2003) 